\title{
The Activation Gate and Gating Mechanism of the NMDA Receptor
}

\author{
Huai-Ren Chang ${ }^{1}$ and Chung-Chin Kuo ${ }^{1,2}$ \\ ${ }^{1}$ Department of Physiology, National Taiwan University College of Medicine, and ${ }^{2}$ Department of Neurology, National Taiwan University Hospital, Taipei \\ 100, Taiwan
}

The NMDA receptor opens in response to binding of NMDA and glycine. However, it remains unclear where and how gating of the NMDA receptor pore is accomplished. We show that different point mutations between S645 and I655 (thus including the highly conserved SYTANLAAF motif) of M3c in NR2B lead to constitutively open channels. The current through these constitutively open channels are readily blocked by external $\mathrm{Mg}^{2+}$ and MK-801 [(+)-5-methyl-10,11-dihydro-5H-dibenzo [a,d] cyclohepten-5,10-imine maleate]. Also, the open-channel blocker MK-801 can no longer be trapped in these channels when NMDA and glycine are washed off. Moreover, M3c residues at or below A651(NR2B, A7 in SYTANLAAF) react with external methanethiosulfonate (MTS) reagents $\sim 500$ to 1000 -fold faster in the presence than in the absence of agonists NMDA and glycine. In fact, the MTS modification rate shows exactly the same NMDA concentration dependence as channel activation. In contrast, those residues external to A651 are always modified with similar kinetics whether NMDA and glycine are present or not. Interestingly, MTS modification of A651C(NR2B) holds the channel constitutively open. Mutations of A651(NR2B) into arginine, tryptophan, or phenylalanine, and similar mutations of the corresponding A652 in NR1 also lead to constitutively open channels. Double-mutant cycle analysis further shows that the effects of A652(NR1) and A651(NR2B) mutations are evidently non-additive (i.e., cooperative) if mutated into residues with large side chains or with compensatory charges [e.g., $\mathrm{A} 652 \mathrm{E}(\mathrm{NR} 1)+\mathrm{A} 651 \mathrm{R}(\mathrm{NR} 2 \mathrm{~B})]$. The side chain of A7 thus plays a determinant role in the intersubunit distance at this level, which is directly responsible for the activation gate and activation- deactivation gating of the NMDA receptor.

Key words: activation gate; constitutive opening; gating mechanism; intersubunit distance; mutant cycle analysis; NMDA receptor

\section{Introduction}

The NMDA receptor opens and closes in response to binding of agonists glutamate (or NMDA) and glycine. Although the agonist-binding domains of the NMDA receptor have been characterized (Kuryatov et al., 1994; Laube et al., 1997; Anson et al., 1998; Furukawa and Gouaux, 2003; Chen et al., 2005; Furukawa et al., 2005), it remains unclear how opening and closing of the pore is accomplished. Each NMDA receptor is composed of four (two NR1 and two NR2) subunits. Each subunit has three transmembrane segments (M1, M3, and M4), a reentrant loop (M2), an extracellular $\mathrm{N}$ terminus [the $\mathrm{N}$-terminal domain (ATD)], and an intracellular $\mathrm{C}$ terminus (for review, see Dingledine et al., 1999; Mayer and Armstrong, 2004). The NMDA channel pore is formed internally by the M2 loop and externally by pre-M1, M3c (the C-terminal part of M3) and M4n (the N-terminal part of M4) from all four subunits (Kuner et al., 1996; Beck et al., 1999; Kashiwagi et al., 2002).

Received Aug. 1, 2007; revised Dec. 17, 2007; accepted Dec. 18, 2007.

This work was supported by Grant NSC 96-2320-B-002-009 from the National Science Council and Grant NHRIEX96-9606NI from the National Health Research Institutes, Taiwan. H.-R.C. was a recipient of the MD-PhD Predoctoral Fellowship from the National Health Research Institutes, Taiwan. We thank Drs. S. Nakanishi and K. Williams for sharing CDNAs encoding NR1a and NR2B subunits.

Correspondence should be addressed to Chung-Chin Kuo, Department of Physiology, National Taiwan University College of Medicine, 1, Jen-Ai Road, 1st Section, Taipei 100, Taiwan. E-mail: chungchinkuo@ntu.edu.tw. DOI:10.1523/JNEUROSCI.3485-07.2008

Copyright $\odot 2008$ Society for Neuroscience $\quad$ 0270-6474/08/281546-11\$15.00/0
The pore region of the NMDA receptor shares evolutionary and structural kinship with an "invertedly" positioned $\mathrm{K}^{+}$channel (Chen et al., 1999; Kuner et al., 1996, 2003; Panchenko et al., 2001). Crystal structures of the KcsA (closed) and MthK (open) $\mathrm{K}^{+}$channels reveal that the inner helices form a gate-like constriction (the bundle crossing) at the intracellular end of the pore (Doyle et al., 1998; Jiang et al., 2002b). Similar localization of the activation gate at the intracellular end of the pore has also been inferred for $\mathrm{Na}^{+}$channels (Kuo and Liao, 2000; Sunami et al., 2004) and $\mathrm{Ca}^{2+}$ channels (Xie et al., 2005). However, the access $\mathrm{of} \mathrm{Ag}^{+}$or methanethiosulfonate (MTS) regents to the pore is not equivalently limited by the bundle crossing in cyclic nucleotidegated and small-conductance $\mathrm{Ca}^{2+}$-activated $\mathrm{K}^{+}$channels (Flynn and Zagotta, 2001; Bruening-Wright et al., 2002), suggesting that the bundle crossing may not form a physical gate in all channels with evolutionary kinship. It is thus interesting to see whether the activation gate of the NMDA receptor has similar location and structural features to Kv channels.

The M3c domain of all glutamate receptor (GluR) subunits contains a highly conserved nine-amino acid motif (SYTANLAAF). The A-to-T mutation at position 8 of this motif (A8T mutation) in the $\delta 2$ glutamate receptor results in constitutive opening of the channel and causes neurodegeneration in the lurcher mutant mouse (Zuo et al., 1997). The T648A (T3A) and A649C (A4C) mutations in NR1 also made constitutively open channels (Kashiwagi et al., 2002; Sobolevsky et al., 2007). The 
M3c domain also shows significant gating conformational changes and thus may play an essential role in activation-deactivation gating of the receptor (Sobolevsky et al., 1999; Kohda et al., 2000; Jones et al., 2002; Kashiwagi et al., 2002; Low et al., 2003; Yuan et al., 2005) (but see Beck et al., 1999; Sobolevsky et al., 2002a,b, 2007). Based on findings that the M3c residues below but not above A651(NR2B, A7 of SYTANLAAF) show statedependent external MTS modification kinetics tightly correlated with channel activation and that the effects of A652(NR1) and A651(NR2B) double mutations are evidently cooperative in holding the channel constitutively open, we conclude that A652(NR1)-A651(NR2B) play a determinant role in the intersubunit configuration at this level, which is directly responsible for the activation-deactivation gating of the NMDA receptor.

\section{Materials and Methods}

Site-directed mutagenesis and expression of NMDA receptors. The rat cDNA clones of NMDA receptors used in this study are the NR1a variant and the NR2B clone. The sequence of amino acid residues in the NR1 and NR2B subunits is numbered from the initiator methionine as in the original papers of NR1 (Moriyoshi et al., 1991) and NR2B (Monyer et al., 1992), respectively. Site-directed mutagenesis was performed using the QuikChange kit (Stratagene, La Jolla, CA). For the preparation of double mutations in the same subunit, cDNA templates that already contained one mutation were used for a second mutation. Mutations were verified by DNA sequencing, and two independent clones for each mutant were tested to preclude any inadvertent mutations. The full-length capped cRNA transcripts were then synthesized from each of NR1 and NR2B using T7 and T3 mMESSAGE mMACHINE transcription kits (Ambion, Austin, TX), respectively. cRNA was stored in aliquots at $-80^{\circ} \mathrm{C}$. To minimize the probability of formation of homomeric NR1 receptors, a mixture of NR1 and NR2 cRNAs in a ratio of 1:5 (i.e., 0.1-4 ng of NR1 and 0.5-20 ng of NR2) (Kashiwagi et al., 2002) was injected into Xenopus oocytes (stages $\mathrm{V}-\mathrm{VI}$ ). Oocytes were maintained in the culture medium (96 mM NaCl, $2 \mathrm{~mm} \mathrm{KCl}, 1.8 \mathrm{~mm} \mathrm{MgCl}_{2}, 1.8 \mathrm{~mm} \mathrm{CaCl}_{2}, 5 \mathrm{~mm}$ HEPES, and $50 \mu \mathrm{g} / \mathrm{ml}$ gentamycin, $\mathrm{pH} 7.6$ ) at $18^{\circ} \mathrm{C}$ for $2-3 \mathrm{~d}$ before electrophysiological recordings. The culture medium was replaced daily.

Electrophysiological studies. Oocytes were placed in a small workingvolume $(<20 \mu \mathrm{l})$ perfusion chamber (OPC-1; AutoMate Scientific, San Francisco, CA) that is optimized for fast solution exchange. The NMDA currents were recorded at a holding potential of $-70 \mathrm{mV}$ and at room temperature $\left(\sim 25^{\circ} \mathrm{C}\right)$ with two-electrode voltage clamp using an OC725C amplifier (Warner Instruments, Hamden, CT). The microelectrodes, pulled from borosilicate glass and filled with $1 \mathrm{M} \mathrm{KCl}$, had resistances of 0.5-4 M $\Omega$. Oocytes were continuously perfused with $\mathrm{Mg}^{2+}$ free ND96 solution ( $96 \mathrm{~mm} \mathrm{NaCl}, 2 \mathrm{~mm} \mathrm{KCl}, 0.3 \mathrm{mM} \mathrm{BaCl}_{2}$, and $5 \mathrm{~mm}$ HEPES, pH 7.6). Solution exchanges were obtained using a pressureregulated, computer-controlled perfusion system (ValveLink 8; AutoMate Scientific). The solution flow rate was $\sim 6 \mathrm{ml} / \mathrm{min}$, and the kinetics of solution exchange were quantified as the following. The NMDA current (elicited by application of $100 \mu \mathrm{M}$ NMDA plus $10 \mu \mathrm{M}$ glycine) was recorded at a holding potential of $-70 \mathrm{mV}$ in $\mathrm{Mg}^{2+}$-free ND96 solution. When the current amplitude reached a steady state, $\mathrm{Na}^{+}$ions were replaced with impermeable $\mathrm{N}$-methyl-D-gluconate (NMG) ions for an instantaneous change in the driving force. The NMDA current then decayed with a two-exponential time course. The fast component had a relative amplitude of $94 \%$ and a time constant of $\sim 0.43 \mathrm{~s}$. The remaining $(6 \%)$ component decayed with a time constant of $\sim 1.4 \mathrm{~s}$. This indicates that $\sim 95 \%$ of solution exchange is completed within $2 \mathrm{~s}$ (assuming that approximately four time constants are enough to "complete" the reaction). NMDA and glycine (Sigma, St. Louis, MO) were dissolved in water, and MK-801 [(+)-5-methyl-10,11-dihydro-5H-dibenzo [a,d] cyclohepten-5,10-imine maleate] (Tocris Biosciences, Bristol, UK) was dissolved in ethanol to make 100, 10, and $10 \mathrm{~mm}$ stock solutions, respectively. The stock solutions were then diluted into $\mathrm{Mg}^{2+}$-free ND96 solution to make $0.1 \mu \mathrm{M}$ to $1 \mathrm{~mm}$ NMDA, $10 \mu \mathrm{M}$ glycine, and $50 \mathrm{~nm}$ or $10 \mu \mathrm{M}$ MK-801 immediate before the experiment. The final concentration of ethanol $(\leq 0.1 \%)$ had no detectable effect on NMDA currents.
Constitutive open index. To investigate macroscopic currents through the constitutively open channel, we obtained currents in the absence and presence of extracellular $\mathrm{Na}^{+}$. As shown in Figure $1 A$, oocytes were voltage clamped at $-70 \mathrm{mV}$ and perfused with $\mathrm{Na}^{+}$-free ND96 solution that contained the impermeable NMG ion ("NMG-ND96": $96 \mathrm{~mm}$ $\mathrm{NMG}, 2 \mathrm{~mm} \mathrm{KCl}, 0.3 \mathrm{~mm} \mathrm{BaCl}$, and $5 \mathrm{~mm}$ HEPES, pH 7.6). The perfusion was then changed to one containing $96 \mathrm{~mm} \mathrm{NaCl}$ ("Na ${ }^{+}-\mathrm{ND} 96$ ": 96 $\mathrm{mm} \mathrm{NaCl}, 2 \mathrm{~mm} \mathrm{KCl}, 0.3 \mathrm{~mm} \mathrm{BaCl}_{2}$, and $5 \mathrm{~mm}$ HEPES, pH 7.6) to elicit the current ("Na ${ }^{+}$-elicited current"). Subsequently, NMDA channel pore blockers $\mathrm{Mg}^{2+}(2 \mathrm{~mm})$ or MK-801 $(10 \mu \mathrm{M})$ were applied with $\mathrm{Na}^{+}$-ND96 solution to the oocytes (to block most, if not all, of the currents). The $\mathrm{Mg}^{2+}$-sensitive $\mathrm{Na}^{+}$-elicited current (the $\mathrm{Na}^{+}$-elicited current that could be blocked by $\mathrm{Mg}^{2+}$ and MK-801 in the absence of NMDA and glycine) is referred to as the NMDA current through the constitutively open NMDA channel. Conversely, oocytes were initially perfused with NMG-ND96 solution and then changed to $\mathrm{Na}^{+}$-ND96 solution containing $100 \mu \mathrm{M}$ NMDA and $10 \mu \mathrm{m}$ glycine to elicit the agonist-elicited NMDA current, which is also readily blocked by $2 \mathrm{~mm}$ $\mathrm{Mg}^{2+}$. In the wild-type and mutant channels, the amplitude of $\mathrm{Mg}^{2+}$ sensitive, $\mathrm{Na}^{+}$-elicited current is divided by that of $\mathrm{Mg}^{2+}$-sensitive "agonist-elicited current" to give the constitutive open index (percentage).

MTS modification. MTS reagents (Toronto Research Chemicals, Toronto, Ontario, Canada) were made as stocks in water everyday under ice-cold condition and mixed into the recording solution within a few minutes before use. Modification rates were determined by either the rate of change in current amplitude during continuous application of MTS reagents (see Fig. 3A) or coapplication of MTS reagents and D-2-amino5-phosphonovaleric acid (APV) $(100 \mu \mathrm{M})$ for different periods of time and documentation of the change in current amplitude (see Fig. $3 B$ ). Application of APV with MTS in this case would help to eliminate the possibility of contamination of the experimental results by any residual glutamate and glycine in the bath solution. The MTS concentration was raised to as high as $3 \mathrm{~mm}$ to measure the slowest rates and lowered to 20 $\mu \mathrm{M}$ to measure the fastest rates. High MTS concentrations could also produce a rapid, reversible reduction (10-20\%) in current size that was also seen in the wild-type channel. This rapid phase was ignored in determining the specific modification rate. The change in current size was fitted with a single-exponential function to obtain the time constant $(\tau)$. The apparent second-order rate constant for MTS modification $(k)$ was given by $k=1 /(\tau[\mathrm{MTS}])$, in which [MTS] is the concentration of the MTS reagent. The rate of solution exchange should be much faster than that of MTS modification itself for an accurate measurement of the modification rate. With our fast perfusion system, MTS concentrations were selected so that $\tau$ was $\sim 10-50$ and $\sim 80-500$ s for the continuous and periodic protocols, respectively. Current rundown was examined for all mutations in the absence of MTS reagents and was always $<10 \%$.

Data analysis. Data were acquired using the Digidata-1322A analog/ digital interface with pClamp software (Molecular Devices, Palo Alto, $\mathrm{CA}$ ), and the sampling rates were $0.5-1 \mathrm{kHz}$. All data are expressed as mean \pm SEM. The Student's $t$ test was used for comparison between experimental groups, and $p<0.05$ was considered as statistically significant.

\section{Results}

\section{Mutations in M3c of the NR2B or the NR1 subunits lead to constitutively open channels}

We made a series of single alanine substitutions in and around $\mathrm{ATD}$, pre-M1, M2, and $\mathrm{M} 3 \mathrm{C}$ of the NR2B subunit (i.e., alanine mutagenesis scan). For ATD, the critical residues for the highaffinity binding site of ifenprodil (a potent gating modifier of the channel) were selected (Perin-Dureau et al., 2002). For M2, residues N615 and N616 were selected. These residues presumably form the narrowest part of the channel pore and are directly responsible for the binding of pore blockers such as $\mathrm{Mg}^{2+}$ and $\mathrm{MK}-801$. For pre-M1 and M3c, most of the residues were selected because these two domains presumably constitute the wall of the external pore mouth and are most likely related to channel gating 
(see Introduction). If the selected residue itself is alanine, it is substituted into nucleophilic cysteine (e.g., A644C, A648C, $\mathrm{A} 651 \mathrm{C}$, and $\mathrm{A} 652 \mathrm{C}$ ). All mutations were screened by the experimental protocol described in Figure $1 A$ (see also Materials and Methods). The mutant receptors that have a significantly different $(p<0.05)$ response from the wild-type receptor are considered to contain mutations at the "key" positions. These key mutations could be approximately correlated with those mutants that had a constitutive open index $>5 \%$ (vs $2 \pm 0.4 \%$ for the wild-type receptor) (Fig. $1 \mathrm{~B}$ ). We found six key positions in the $46 \mathrm{NR} 2 \mathrm{~B}$ mutations tested (all of which generated functional receptors, except F554A, W559A, N649A, and F653A). Interestingly, these six key mutations are clustered in two regions, namely M3c (S645A, T647A, A648C, and I655A) and pre-M1 (S555A and D557A), both being regions likely to line the pore. These results are consistent with the idea that $\mathrm{M} 3 \mathrm{c}$ and pre-M1 are critically related to channel gating. Because the two positions in pre-M1 (S555 and D557) of NR2A were not substantially modified by external MTS reagents regardless of whether the agonists are present or not (Thomas et al., 2006), we would focus on the key positions in M3c. NMDA receptors are heterotetrameric receptors composed of the NR1 and NR2 subunits, both contributing to the formation of the channel pore. We therefore further examined the constitutive open index in NR1 mutant receptors. Very much analogous to the observation in the homologous residues in NR2B, T648A, A649C, V656A, and L657A mutations in NR1 also lead to constitutively open channels (Fig. $1 B$ ).

MK-801 can no longer be trapped in the constitutively open channels

It has been shown that externally applied MK-801, an open channel blocker of the NMDA receptor, can be "trapped" behind the closed activation gate after dissociation of agonists from the receptor (Huettner and Bean, 1988). In other words, external MK-801 can enter the open NMDA channel and bind to the "blocking site" located deep in the pore (probably very close to the selectivity filter) (Kashiwagi et al., 2002). Occupancy of this site, however, does not prevent channel closure, and the blocking molecule can be kept behind the closed activation gate for a long time. Conversely, subsequent openings of the activation gate would greatly facilitate the trapped blocker to leave the channel (Antonov et al., 1998; Sobolevsky et al., 1999; Qian and Johnson, 2002). More recently, it was also proposed that MK-801 and other trapping channel blockers probably bind close to and interact with the activation-deactivation gating machinery of the
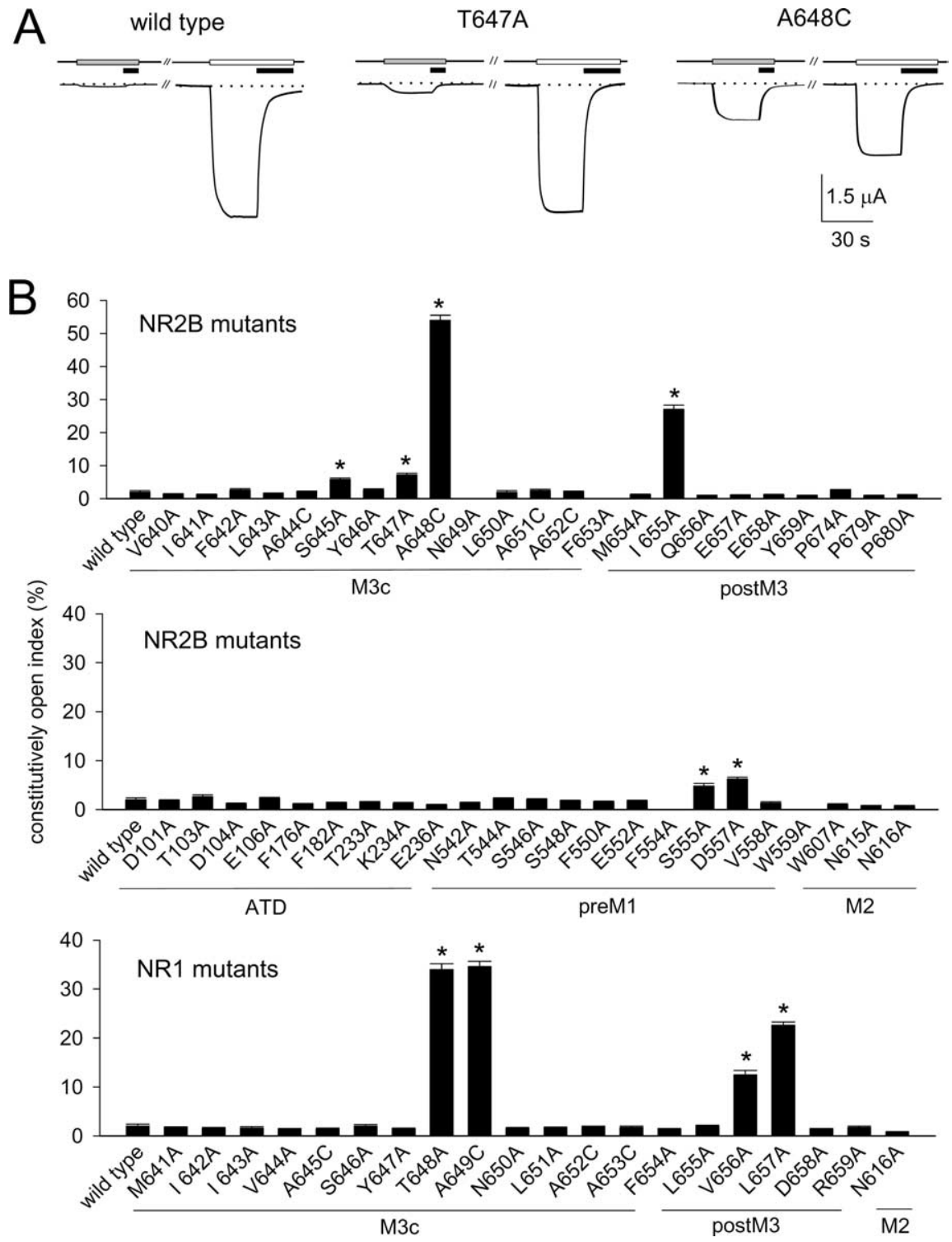

Figure 1. Point mutations generating constitutively open channels. $A$, The oocytes coexpressing wild-type NR1 with either the wild-type or mutant NR2B subunits were perfused with $\mathrm{Mg}^{2+}$-free NMG-ND96 solution (thick line), and then the perfusion was changed to $\mathrm{Mg}^{2+}$-free $\mathrm{Na}^{+}{ }_{-} \mathrm{ND} 96$ solution (the gray bar) to elicit the $\mathrm{Na}^{+}$-elicited current. $\mathrm{Mg}^{2+}$ at $2 \mathrm{~mm}$ (black bar) was then applied with $\mathrm{Na}^{+}{ }^{+} \mathrm{ND} 96$ solution to the 00 cytes for 10 s to block the current. Conversely, the same 00 cytes were also perfused with $\mathrm{Mg}^{2+}$-free NMG-ND96 solution and then changed to $\mathrm{Mg}^{2+}$-free $\mathrm{Na}^{+}-\mathrm{ND} 96$ solution containing $100 \mu \mathrm{MM}$ NMDA and 10 $\mu \mathrm{m}$ glycine (white bar) to elicit the agonist-elicited current, which is again blocked by $2 \mathrm{~mm} \mathrm{Mg}^{2+}$ (black bar). The amplitude of $\mathrm{Mg}^{2+}$-sensitive, $\mathrm{Na}^{+}$-elicited current is divided by that of $\mathrm{Mg}^{2+}$-sensitive, agonist-elicited current to give the constitutive open index of 2,7.8, and 50\% for the wild-type, T647A, and A648C mutant receptors, respectively. In contrast, there is no discernible $\mathrm{Na}^{+}$- or agonist-elicited current in control oocytes that are not expressing NMDA receptor subunits $(n=6$; data not shown). The dotted lines indicate zero current level. $\boldsymbol{B}$, Cumulative results are obtained with the same experimental protocol described in $\boldsymbol{A}$ ( $n=10-12$ for each different channel). The constitutive open index is calculated and summarized for NR2B mutant receptors (top and middle) and NR1 mutant receptors (bottom). For N615A, N616A (NR2B), and N616A (NR1) mutations, $2 \mathrm{~mm} \mathrm{Mg}^{2+}$ had a negligible inhibitory effect on the current, and the index was calculated directly using the amplitude of $\mathrm{Na}^{+}$-elicited current divided by that of agonist-elicited current. ${ }^{*} p<0.05$, compared with the data of the wild-type receptor.

NMDA receptor (Yuan et al., 2005; Dravid et al., 2007). MK-801 therefore could be a very useful probe to detect whether the activation gate of the NMDA receptor is closed or open. We examined recovery from block by MK-801 in the wild-type and selected NR2B mutant receptors. Figure 2 shows that recovery from MK-801 block of the wild-type receptor in the absence of agonists is very slow, with a time constant of $\sim 20 \mathrm{~min}$, consistent with the idea of trapped MK-801 in the closed channel (Huettner and Bean, 1988). In contrast, T647A mutation speeds the recovery 
A

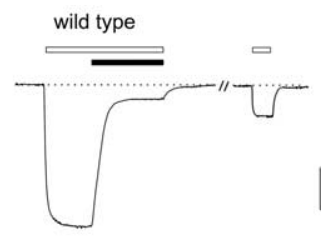

$\mathrm{B}$
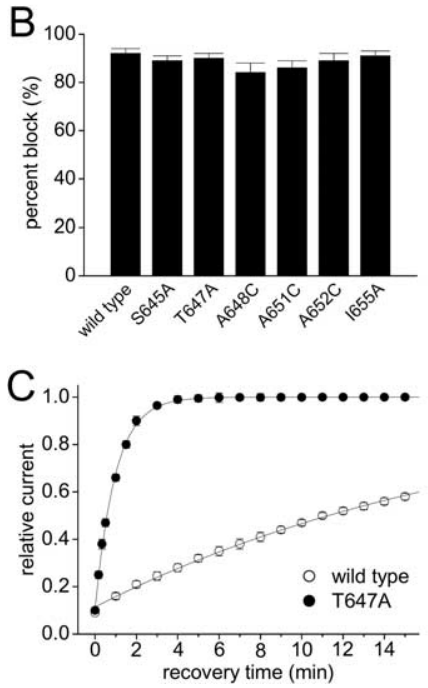

Figure 2. Recovery from block by MK-801. A, NMDA currents obtained from oocytes coexpressing wild-type NR1 and either the wild-type or mutant NR2B subunits were elicited by application of $100 \mu \mathrm{m}$ NMDA with $10 \mu \mathrm{m}$ glycine. For the following $60 \mathrm{~s}, 50 \mathrm{~nm}$ MK-801 was applied with agonists (NMDA and glycine) to block the NMDA current. The oocyte was then washed with control ND96 solution for $120 \mathrm{~s}$ (recovery time) before a final NMDA pulse was given for $15 \mathrm{~s}$. The white and black bars above the current traces indicate application of agonists and MK-801, respectively. The dotted lines indicate zero current level. $\boldsymbol{B}$, Cumulative results are obtained with the same experimental protocol described in $\boldsymbol{A}$ ( $n=6-8$ for each different channel). Left, The block of the NMDA current by $50 \mathrm{~nm}$ MK-801 is not significantly different among the wild-type and different mutant receptors ( $p>0.05)$. Right, The current amplitude in the final NMDA pulse is normalized to that in the first control NMDA pulse (before MK-801 block) to give the fraction recovered. The S645A, T647A, A648C, and I655C, but not A651C and A652C, mutant receptors show significantly higher fraction recovered than the wild-type recep$\operatorname{tor}\left({ }^{*} p<0.05\right)$. C, The recovery course from MK-801 block is obtained by a two-pulse protocol with a progressively lengthened interpulse (recovery) interval ( $0-15 \mathrm{~min})$. The fraction recovered is plotted against duration of the recovery time. The lines are fits with single-exponential functions, giving time constants of $\sim 20 \mathrm{~min}$ and $59 \mathrm{~s}$ for the wild-type and T647A mutant receptors, respectively (both $n=6-8$ ).

course with shortening of the time constant to $\sim 60 \mathrm{~s}$ (Fig. $2 C$ ). This result indicates that MK-801 cannot be trapped in T647A mutant receptor after dissociation of agonists from the receptor. Moreover, similar findings can be obtained in the other constitutively open mutants (e.g., S645A, A648C, and I655A) but not in the nonconstitutively open mutants (e.g., A651C and A652C) (Fig. 2B). These results support that these constitutively open channels indeed have an open activation gate.

The inner but not the outer part of M3c (NR2B) shows statedependent accessibility to external MTS reagents

Figure $3 A$ shows agonist-elicited NMDA currents recorded in Xenopus oocytes expressing cysteine-substituted NR2B mutant (V640-Q656) receptors. Application of external 2-aminoethylmethanethiosulfonate (MTSEA) typically reduces current amplitude [e.g., T647C and M654C (Fig. 3A); V640C, L643C, A648C, I655C and Q656C (data not shown)]. However, MTSEA increases rather than decreases the currents in two instances, namely A651C and A652C (Fig. 3A). For all cysteine-substituted mutant receptors, changes in the current amplitude with application of MTSEA could be well fitted with single-exponential functions. The apparent second-order rate constants (i.e., $k$ value in the equation in Materials and Methods) for MTS modification in the presence of agonists (i.e., when the receptor is predominantly distributed into the "activated" states, which presumably include both the conducting open and the nonconducting desensitized conformations) are derived from the time constants from the fits and are $889,379,769$, and $833 \mathrm{M}^{-1} \mathrm{~s}^{-1}$ for T647C, A651C, A652C, and M654C mutant receptors, respectively (Fig. 3A). We further measured the modification rate of cysteine-substituted NR2B mutant receptors in the absence of agonists (i.e., when the receptor is predominantly distributed into the closed state). Four typical examples of the apparent second-order rate constants derived from the time constants from the single-exponential fits to the courses of modification are $1.86,<0.83,49$, and $204 \mathrm{M}^{-1} \mathrm{~s}^{-1}$ for T647C, A651C, A652C, and M654C mutant receptors, respectively (Fig. $3 B$ ). Figure 3, $C$ and $D$, summarizes the pattern of state-dependent reactivity of single cysteine substitution in M3c and M2 based on experiments similar to the preceding ones. The substituting cysteines at positions N615 and N616 in M2, and V640, L643, T647 and A651 in M3c are modified almost exclusively in the activated state, with at least an $\sim 500$ - to 1000 -fold faster rate of modification in the activated than in the closed state. These results are consistent with the previous reports that N616C, V644C, T648C, and A652C of the NR1 subunit (homologous to N615, L643, T647, and A651 of NR2B, respectively) show significant state-dependent reaction kinetics with external MTSEA (Jones et al., 2002; Sobolevsky et al., 2002a; Yuan et al., 2005). Conversely, residues above T647-A651 (i.e., positions A652-Q656) show much smaller reactivity changes associated with channel gating (at most an approximately threefold to fivefold preference for the activated state). This overall pattern leads us to hypothesize that access of MTS reagents to the deeper region (N615-N616 in M2 and V640-T647 in M3c) of the pore is controlled by the activation gate of the channel, very much similar to the way how the access of traveling ions (e.g., $\mathrm{Na}^{+}$) to the pore is regulated. At positions M654-Q656 in NR2B, the access does not seem to be controlled by the activation gate. Instead, there is probably a gating-associated movement that results in small (approximately threefold to fivefold) changes in side-chain accessibility. Small changes in cysteine reactivity associated with gating have also been observed in the lower part of S6 of the Shaker $\mathrm{K}^{+}$ channel (Liu et al., 1997).

\section{MTS modification rate shows exactly the same NMDA concentration dependence as channel activation}

To confirm that the marked state dependence of modification rates was indeed ascribable to channel gating, we characterized the NMDA concentration dependence of 2-trimethylammonioethyl-methanethiosulfonate (MTSET) modification in detail in the L643C(NR2B) mutant receptor (i.e., L643 is presumably a pore-lining residue located below T647-A651). Figure 4 shows that the second-order rate constants obtained at different NMDA concentrations are well superimposable on the NMDA channel activation (opening probability) curve. It is clear that the modification rate rises steeply with the increasing NMDA concentration in parallel with channel gating (the NMDA channel activation curve) and reaches a plateau value at the NMDA concentration giving the maximum current. The perfect superposition of the two curves is reasonable considering the similar diameters of the smallest hydrated $\mathrm{Na}^{+}$ion $(\sim 7 \AA)$ and MTSEA, MTSET, and MK-801 ( 3.6, 5.8, and $\sim 7.2 \AA$, respectively). 

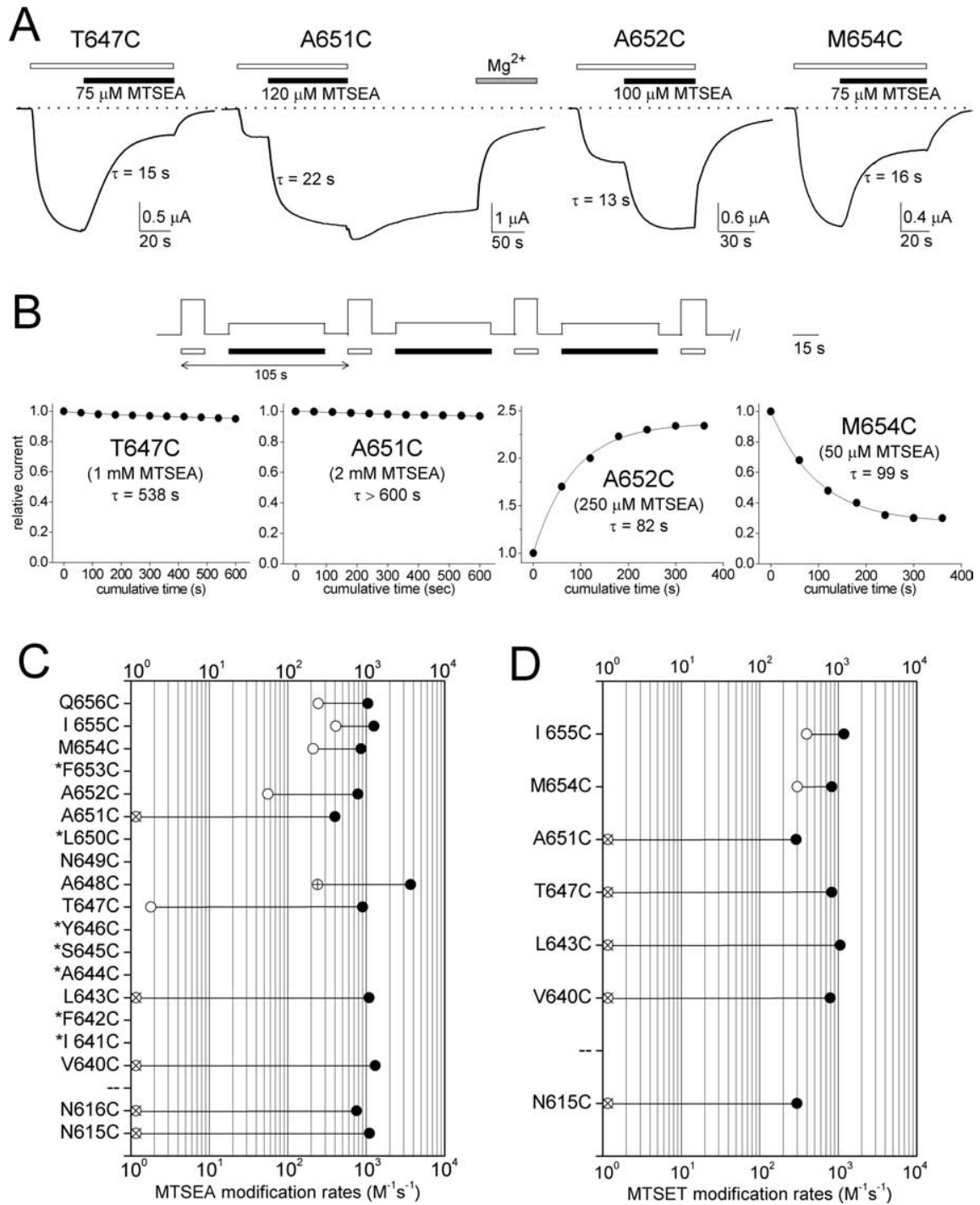

Figure 3. State-dependent accessibility of substituted cysteines in M3c of the NR2B subunit. $A$, NMDA currents obtained from oocytes coexpressing wild-type NR1 and the cysteine substituted NR2B subunits were elicited by application of $100 \mu \mathrm{m} \mathrm{NMDA}$ and $10 \mu \mathrm{m}$ glycine. MTSEA was then applied with agonists (NMDA and glycine) to the 0ocytes for $\sim 60-120 \mathrm{~s}$. The white and black bars above the current traces indicate application of agonists and MTSEA, respectively. The kinetics of current amplitude changes (either decrease or increase) during MTSEA application are reasonably fitted with single-exponential functions, giving time constants of $15,22,13$, and $16 \mathrm{~s}$ for $\mathrm{T} 647 \mathrm{C}, \mathrm{A} 651 \mathrm{C}, \mathrm{A} 652 \mathrm{C}$, and $\mathrm{M} 654 \mathrm{C}$ mutant receptors, respectively. Conversely, the change in maximal currents is $-78,311,126,-65 \%$ for T647C, $A 651 C, A 652 C$, and M654C mutant receptors, respectively. The decrease of current after modification in T647C and M654C are typical of the other mutant receptors (N615C, N616C, V640C, L643C, A648C, 1655C, and Q656C), whereas the increase of current after modification is characteristic for $\mathrm{A} 651 \mathrm{C}$ and $\mathrm{A652C}$ mutant receptors. It is also evident that modification of A651C mutant receptor with MTSEA not only potentiates the NMDA current but also holds the channel open after removal of agonists. This persistent current in the absence of agonists is effectively blocked by $2 \mathrm{~mm} \mathrm{Mg}^{2+}$ (gray bar) but is not affected by APV (data not shown). The dotted lines indicate zero current level. $\boldsymbol{B}$, Measurement of the modification rates of MTSEA in the absence of agonists. Every $105 \mathrm{~s}$, NMDA currents were elicited by application of $100 \mu \mathrm{m}$ NMDA plus $10 \mu \mathrm{m}$ glycine (NMDA pulse) for $15 \mathrm{~s}$. Between two brief (15 s) NMDA pulses, MTSEA and APV (100 $\mu \mathrm{m}$ ) was coapplied for $60 \mathrm{~s}$ with both a preceding and a following $15 \mathrm{~s}$ NMDA-free MTSEA-free washout pulses. The white and black bars indicate application of agonists and MTSEA + APV, respectively. The relative current is plotted against the cumulative time of MTSEA application and is fitted with a single-exponential function, giving time constants of 538, >600, 82, and $99 \mathrm{~s}$ for T647C, A651C, A652C, and M654C mutant receptors, respectively. Note that A652C could also be evidently modified by MTSEA in the absence of agonists, but it needs higher concentration and longer exposure time than that in the presence of agonists (Fig. $3 \mathrm{~A}$ ). We also measured the MTSEA modification rates in the absence of agonists by the other protocol (without coapplication of APV when MTSEA is present; data not shown). In general, there are only negligible (less than $\sim 10 \%$ ) differences of the modification rates between the former protocol (coapplication of MTS and APV) and the latter protocol (MTS alone without APV). The negligible differences between these two protocols argue that there are little contamination by any residual glutamate and glycine in our experimental system and that the agonist affinity does not seem to increase significantly in these mutant receptors. C, Second-order rate constants are plotted for MTSEA modification of cysteines in the absence (open circles) or presence (filled circles) of agonists. Positions marked with an asterisk are not substantially affected by MTSEA (i.e., like the wild-type receptor). Modification rates for A651C, L643C, V640C, N616C, and N615C in the absence of agonists $(\otimes)$ could not be determined properly because they required very high concentrations of MTSEA (approximately $\geq 2 \mathrm{~mm}$ ), which may then have nonspecific effects on the membrane or
The tight correlation between the gated access of external MTSET to the substituted cysteine and the gated $\mathrm{Na}^{+}$permeation through the pore strongly argues that both processes are controlled by the same gate of the channel.

\section{Modification of A651C(NR2B) with external MTS reagents holds the channel constitutively open}

We have demonstrated that A651C(NR2B) mutant receptor shows remarkably state-dependent reaction kinetics with external MTS reagents (Fig. 3C,D). The effect of MTS modification on A651C(NR2B) mutant receptor, however, is quite different from the other tested residues. Modification of A651C(NR2B) with external MTS reagents happens essentially only in the presence of agonists and then would tend to hold the channel open. MTS modification therefore enhances the NMDA current. It is already evident in Figure $3 A$ that, after MTESA modification, the channel allows a much larger inward current and remains open even after removal of agonists. Subsequent application of $\mathrm{Mg}^{2+}$ (an open channel blocker) (Fig. 3A) but not APV (a competitive NMDA site antagonist; data not shown) blocks this constantly open channel. Moreover, modification of homologous $\mathrm{A} 652 \mathrm{C}(\mathrm{NR} 1)$ by external MTSET shows essentially the same findings (data not shown) (Jones et al., 2002; Yuan et al., 2005). Because of the structural similarity between MTS-modified cysteine and arginine (Fig. 5A), we examined arginine substitutions in A651(NR2B) and homologous A652(NR1) to see whether they also make constitutively open channels. Figure $5 B$ shows that there are very similar current amplitude and activation kinetics between the $\mathrm{Na}^{+}$-elicited current and agonist-elicited current (or more precisely "agonist- and $\mathrm{Na}^{+}$-elicited current" because, in this case, the external solution was switched from agonist-free NMG-ND96 to agonistcontaining $\left.\mathrm{Na}^{+}-\mathrm{ND} 96\right)$ in $\mathrm{A} 651 \mathrm{R}(\mathrm{NR} 2 \mathrm{~B})$ mutation. The constitutive open index is $96 \pm 2.2$ and $71 \pm 1.7 \%$ for A651R(NR2B) and $\mathrm{A} 652 \mathrm{R}(\mathrm{NR} 1)$ mutations, respectively

\section{$\leftarrow$}

receptors. These rates are smaller than $1 \mathrm{M}^{-1} \mathrm{~S}^{-1}$ but are assigned with this value for simplicity. Each point is the mean of five or more determinations, and SEs are all smaller than the symbols. A648C mutation (D) generates a constitutively open channel in the absence of agonists (and cannot be blocked by APV; see also Fig. 1), but the other cysteine substituting mutations do not. N649C mutation does not express functional channels $(n=16)$. $\boldsymbol{D}$, The same experiment as that described in Cwas repeated in selected residues, but the modifying MTS reagent was MTSET rather than MTSEA. 


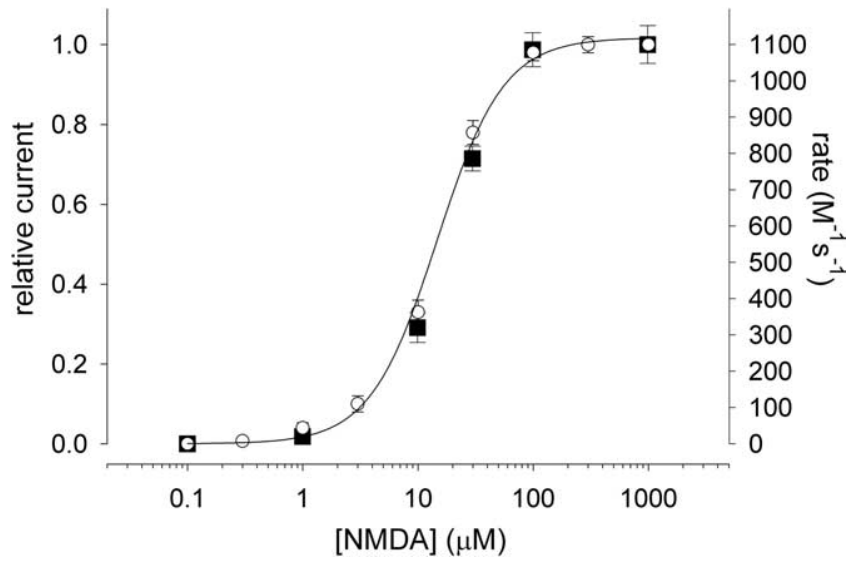

Figure 4. NMDA concentration dependence of the MTSET modification rate of $L 643$ (NR2B) mutant receptor. NMDA concentration-response curves for L643C(NR2B) mutant receptor are constructed in the presence of a saturating concentration of glycine $(10 \mu \mathrm{M})$. For comparison, graphs are shown for relative NMDA currents (open circles, left vertical axis) and MTSET modification rates (filled squares, right vertical axis) (both $n=5-8$ ). The solid line (i.e., NMDA channel activation curve) is the fit for the data points of relative NMDA currents using the Hill equation $I=I_{\max } /\left(1+\left(K_{\text {app }} /[\mathrm{NMDA}]\right)^{n H}\right)$, where $I$ is the experimental current in a given concentration of NMDA, $I_{\max }$ is the maximum current, [NMDA] is the NMDA concentration, $K_{\text {app }}$ is the apparent affinity of NMDA to the channel, and $n_{H}$ is the Hill coefficient. The $K_{\text {app }}$ and $n_{H}$ values are $13.6 \mu \mathrm{m}$ and 1.3 from the fit, respectively. The same fitting curve could also reasonably describe the NMDA concentration dependence of the modification kinetics. For example, in the presence of $0.1 \mu \mathrm{M} \mathrm{NMDA}$, when $\sim 99 \%$ of the channels are closed, modification is extremely slow. In the presence of $30 \mu \mathrm{M} N \mathrm{NMA}$, when $\sim 80 \%$ of the channels are open, modification is $\sim 800$-fold faster that that in the presence of $0.1 \mu \mathrm{m} \mathrm{NMDA}$. This large increase in the modification rate is apparently a consequence of channel activation. The modification rate increases by only $\sim 1.3$-fold from 30 to $1000 \mu \mathrm{MNMDA}$, however, when the magnitude of the NMDA current also reaches a plateau. This small increase also indicates that MTSET diffusion into the pore itself has little intrinsic NMDA concentration dependence.

(both $n=10$ ) (Table 1). The fact that the presence of agonists (agonist-elicited current) or not $\left(\mathrm{Na}^{+}\right.$-elicited current) does not cause a discernible difference in the elicitation of the NMDA currents is consistent with the foregoing view that A651C(NR2B) + MTS already holds the channel constitutively open. Similar to the wild-type channel, the $\mathrm{Na}^{+}$-elicited currents of these two constitutively open channels are readily blocked by $\mathrm{Mg}^{2+}$ and MK-801. Most interestingly, N615C(NR2B), a residue presumably located deep in the pore (i.e., at the tip of M2 loop) and responsible for $\mathrm{Mg}^{2+}$ as well as $\mathrm{Ca}^{2+}$ binding, can be modified by external MTSET in the A651R+N615C(NR2B) double mutation in the complete absence of agonists (Fig. $5 C$ ). Moreover, the modification kinetics of MTSET are very similar in single-mutant N615C(NR2B) in the presence of agonists (the N615C mutant receptor is not constitutively open and cannot be modified by MTSET in the absence of agonists) and doublemutant $\mathrm{A} 651 \mathrm{R}+\mathrm{N} 615 \mathrm{C}(\mathrm{NR} 2 \mathrm{~B})$ in the absence of any agonists $\left(k=298 \pm 21\right.$ and $267 \pm 18 \mathrm{M}^{-1} \mathrm{~s}^{-1}$, respectively; $p>0.05 ; n=$ 8) (Fig. 5C). These results strongly suggest that A651R(NR2B) mutation leads to a constitutively open channel with an activation gate conformation very similar to that of the open wild-type channel (and also with essentially unaltered functional properties of the pore concerning ion permeation or $\mathrm{Mg}^{2+}$ and $\mathrm{MK}-801$ block).

Double-mutant cycle analysis shows cooperative interaction between homologous residues A652(NR1) and A651(NR2B) Because A652(NR1) and A651(NR2B) seem to play a unique role in NMDA channel gating, we further investigated the possible interactions between these two homologous residues to elucidate
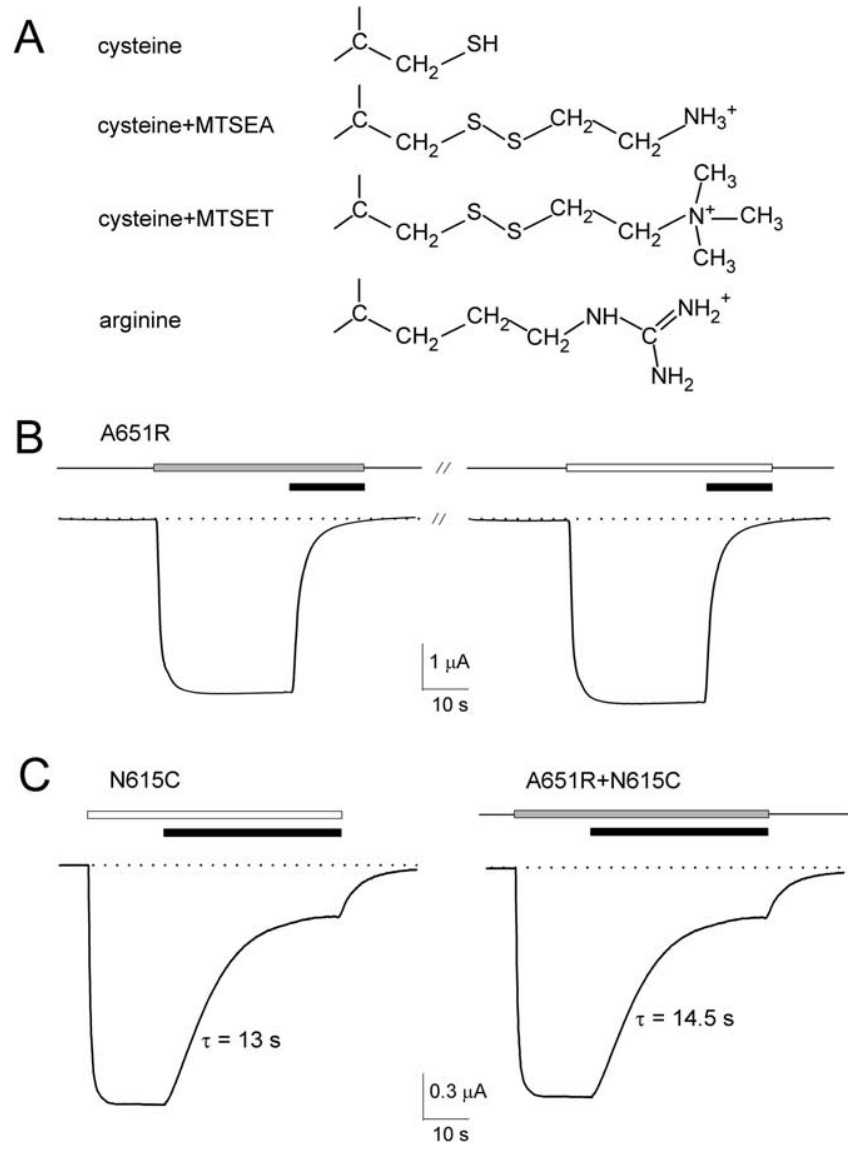

Figure 5. A651R(NR2B) single mutation leading to nearly fully constitutively open channels. $A$, Schematic structures of cysteine with and without modification by MTSEA or MTSET. A positively charged arginine residue is shown for comparison. $\boldsymbol{B}$, The same experiment as that described in Figure $1 A$ was repeated in A651R(NR2B) mutant receptor. The thick line, gray bar, white bar, and black bar represent application of NMG-ND96, agonist-free $\mathrm{Na}^{+}-\mathrm{ND} 96, \mathrm{Na}^{+}{ }_{-}$ ND96 plus agonists (100 $\mu \mathrm{m}$ NMDA and $10 \mu \mathrm{m}$ glycine), and $2 \mathrm{~mm} \mathrm{Mg}^{2+}$, respectively. The dotted lines indicate zero current level. It is evident that A651R mutant receptor has a large $\mathrm{Na}^{+}$-elicited current, which can be blocked by $\mathrm{Mg}^{2+}$ (left). Conversely, the current amplitude and kinetics of $\mathrm{Mg}^{2+}$ block of the agonist-elicited current (right) in A651R(NR2B) mutant receptor are very similar to those of the $\mathrm{Na}^{+}$-elicited current. The constitutive open index is $95 \%$ in this case. C, MTSET modification of single-mutant N615C(NR2B) and double-mutant $A 651 R+N 615 C$ (NR2B) receptors was studied with the same experimental protocol as that described in Figure $3 A$. The thick line, gray bar, white bar, and black bar represent application of NMG-ND96, agonist-free $\mathrm{Na}^{+}{ }^{+}$-ND96, $\mathrm{Na}^{+}{ }^{+}$-ND96 plus agonists (100 $\mu \mathrm{M}$ NMDA and $10 \mu \mathrm{M}$ glycine), and MTSET (250 $\mu \mathrm{M})$, respectively. In N615C(NR2B) single mutation, MTSET inhibits the agonist-elicited current by $80 \%$ with a current decay time constant of $13 \mathrm{~s}$ (left). MTSET has no effect on this channel if no agonists are present (data not shown). Conversely, A651R + N615C(NR2B) double mutation led to a constitutively open channel. MTSET inhibits the $\mathrm{Na}^{+}$-elicited current by $78 \%$ with a decay time constant of $14.5 \mathrm{~s}$ in the absence of any agonist (right).

the molecular mechanisms underlying the control of the activation gate. If the difference in free energy $(\Delta G)$ between closed and activated NMDA receptors is simplistically calculated according to the Boltzmann equation as follows: $\Delta G=R T \ln (A / C)$, where $R$ is the gas constant, $T$ is the absolute temperature, and $A / C$ is the ratio between the steady-state occupancy of the activated states (open plus desensitized states) and the closed state of the NMDA receptor. Assuming a constant ratio between the occupancies of the open and desensitized states and nearly complete occupancy of the receptor in the activated states (i.e., scarcely any receptor in the resting state) when $100 \mu \mathrm{M}$ NMDA/10 $\mu \mathrm{M}$ glycine is present, we may readily obtain $A / C$ from the ratio between constitutive 
Table 1. Double-mutant cycle analysis of A652(NR1) and A651(NR2B) mutations

\begin{tabular}{|c|c|c|c|c|}
\hline & $\begin{array}{l}\text { Constitutive } \\
\text { open index (\%) }\end{array}$ & $\begin{array}{l}\Delta G \\
\text { (kcal/mol) }\end{array}$ & $\begin{array}{l}\Delta \Delta G \\
(\mathrm{kcal} / \mathrm{mol})\end{array}$ & $\begin{array}{l}\Delta \Delta G_{\text {int }} \\
\text { (kcal/mol) }\end{array}$ \\
\hline Wild type & $2.0 \pm 0.4$ & $-2.35 \pm 0.02$ & & \\
\hline A652E(NR1) & $25 \pm 1.2$ & $-0.67 \pm 0.01$ & $1.68 \pm 0.03$ & \\
\hline A651R(NR2B) & $96 \pm 2.2$ & $1.91 \pm 0.08$ & $4.26 \pm 0.07$ & \\
\hline $\mathrm{A} 652 \mathrm{E}(\mathrm{NR} 1)+\mathrm{A} 651 \mathrm{R}(\mathrm{NR} 2 \mathrm{~B})^{a}$ & $65 \pm 1.1$ & $0.37 \pm 0.01$ & $2.72 \pm 0.03$ & $-3.22 \pm 0.06$ \\
\hline A652R(NR1) & $71 \pm 1.7$ & $0.54 \pm 0.01$ & $2.89 \pm 0.02$ & \\
\hline A651E(NR2B) & $15 \pm 0.3$ & $-1.03 \pm 0.02$ & $1.32 \pm 0.03$ & \\
\hline $\mathrm{A} 652 \mathrm{R}(\mathrm{NR} 1)+\mathrm{A} 651 \mathrm{E}(\mathrm{NR} 2 \mathrm{~B})^{a}$ & $30 \pm 0.7$ & $-0.50 \pm 0.01$ & $1.85 \pm 0.02$ & $-2.36 \pm 0.04$ \\
\hline A652D(NR1) & $69 \pm 0.9$ & $0.48 \pm 0.01$ & $2.83 \pm 0.03$ & \\
\hline A651K(NR2B) & $90 \pm 1.6$ & $1.32 \pm 0.02$ & $3.67 \pm 0.02$ & \\
\hline A652D(NR1) + A651K(NR2B) & $85 \pm 1.2$ & $1.04 \pm 0.01$ & $3.39 \pm 0.03$ & $-3.11 \pm 0.09$ \\
\hline A652K(NR1) & $79 \pm 1.1$ & $0.79 \pm 0.01$ & $3.14 \pm 0.03$ & \\
\hline A651D(NR2B) & $93 \pm 1.7$ & $1.55 \pm 0.02$ & $3.90 \pm 0.005$ & \\
\hline A652K(NR1) + A651D(NR2B) & $90 \pm 1.8$ & $1.32 \pm 0.02$ & $3.67 \pm 0.03$ & $-3.37 \pm 0.06$ \\
\hline A652C(NR1) & $2.4 \pm 0.4$ & $-2.21 \pm 0.01$ & $0.14 \pm 0.01$ & \\
\hline A651C(NR2B) & $1.8 \pm 0.2$ & $-2.41 \pm 0.01$ & $-0.06 \pm 0.01$ & \\
\hline$A 652 C(N R 1)+A 651 C(N R 2 B)$ & $0.8 \pm 0.1$ & $-2.90 \pm 0.03$ & $-0.55 \pm 0.02$ & $-0.63 \pm 0.02$ \\
\hline A652T(NR1) & $1.8 \pm 0.2$ & $-2.41 \pm 0.05$ & $-0.06 \pm 0.02$ & \\
\hline A651T(NR2B) & $2.2 \pm 0.3$ & $-2.26 \pm 0.03$ & $0.09 \pm 0.02$ & \\
\hline A652T(NR1) + A651T(NR2B) & $0.9 \pm 0.1$ & $-2.83 \pm 0.06$ & $-0.48 \pm 0.04$ & $-0.45 \pm 0.01$ \\
\hline A652F(NR1) & $0.9 \pm 0.2$ & $-2.83 \pm 0.07$ & $-0.48 \pm 0.06$ & \\
\hline A651F(NR2B) & $1.1 \pm 0.3$ & $-2.71 \pm 0.05$ & $-0.36 \pm 0.04$ & \\
\hline $\mathrm{A} 652 \mathrm{~F}(\mathrm{NR} 1)+\mathrm{A} 651 \mathrm{~F}(\mathrm{NR} 2 \mathrm{~B})^{b}$ & $46 \pm 1.4$ & $-0.10 \pm 0.01$ & $2.25 \pm 0.03$ & $3.09 \pm 0.06$ \\
\hline A652L(NR1) & $0.8 \pm 0.2$ & $-2.90 \pm 0.07$ & $-0.55 \pm 0.02$ & \\
\hline A651L(NR2B) & $1.2 \pm 0.2$ & $-2.65 \pm 0.06$ & $-0.30 \pm 0.02$ & \\
\hline$A 652 L(N R 1)+A 651 L(N R 2 B)^{b}$ & $56 \pm 1.9$ & $0.14 \pm 0.02$ & $2.49 \pm 0.01$ & $3.34 \pm 0.07$ \\
\hline A652W(NR1) & $10 \pm 0.5$ & $-1.33 \pm 0.04$ & $1.02 \pm 0.02$ & \\
\hline A651W(NR2B) & $30 \pm 1.1$ & $-0.50 \pm 0.01$ & $1.85 \pm 0.02$ & \\
\hline $\mathrm{A} 652 \mathrm{~W}(\mathrm{NR} 1)+\mathrm{A} 651 \mathrm{~W}(\mathrm{NR2B})^{b}$ & $97 \pm 2.1$ & $2.09 \pm 0.07$ & $4.44 \pm 0.08$ & $1.57 \pm 0.04$ \\
\hline
\end{tabular}

$\Delta G$, The difference in free energy between closed and activated channels; $\Delta \Delta G$, the change in $\Delta G$ caused by each mutation; $\Delta \Delta G_{\text {int, }}$ the coupling energy (for more details, see Results).

${ }^{a}$ Double mutations showing a significantly $(p<0.05)$ smaller constitutive open index than one of the two component single mutations.

${ }^{b}$ Double mutations showing a significantly ( $p<0.05$ ) larger constitutive open index than the addition of the two component single mutations (data are expressed as mean \pm SEM; $n=8-12$ for each mutation).

open index and $(1-$ constitutive open index) for each different receptor. The change in $\Delta G$ caused by each mutation $(\Delta \Delta G)$ could be calculated as follows: $\Delta \Delta G=\Delta G_{\text {mut }}-\Delta G_{\mathrm{wt}}$. To differentiate whether two mutations interact independently or cooperatively in channel gating, we used double-mutant cycle analysis (Hidalgo and MacKinnon, 1995) to calculate the coupling energy $\left(\Delta \Delta G_{\text {int }}\right)$ for each pair of mutations. If the two residues are coupled in energy terms, there will be a coupling energy $\Delta \Delta G_{\text {int }}$ given by $\Delta \Delta G_{\text {int }}=\Delta \Delta G_{A 12}-\left(\Delta \Delta G_{A 1}+\Delta \Delta G_{A 2}\right)$, where $A_{1}$ and $A_{2}$ are single mutations, and $A_{12}$ is the double mutation $A_{1}+A_{2}$. Table 1 shows that different charged single mutations (e.g., A to R, E, K, or D) of these two homologous residues, A652(NR1) and A651(NR2B), lead to channels with constitutive open indexes of 15-96\%. Most interestingly, A652R(NR1)+A651E(NR2B) and $\mathrm{A} 652 \mathrm{E}(\mathrm{NR} 1)+\mathrm{A} 651 \mathrm{R}(\mathrm{NR} 2 \mathrm{~B})$ have a much smaller constitutive open index than single mutations A652R(NR1) and A651R(NR2B), suggesting a cooperative (non-additive) action between these two homologous residues. Moreover, the significant negative coupling energies $\left(\Delta \Delta G_{\text {int }}=-2.36\right.$ to $-3.37 \mathrm{kcal} /$ $\mathrm{mol}$ ) (Fig. 6A) further indicate that there is a significant direct interaction between these two homologous compensatorycharge mutant residues in NR1 and NR2B. These findings strongly argue that A652(NR1) and A651(NR2B) may play a critical role in setting the configuration of the activation gate. We therefore also explored the steric or voluminal effect of the residues at this critical position. Single and double mutations by substituting A652(NR1) and A651(NR2B) with residues of "medium" side chains (e.g., cysteine or threonine, in contrast with the "small"-size alanine in the wild-type channel) cannot make constitutively open channels (Fig. 6B, Table 1). Although single mu- tations to "large" side chains (e.g., leucine or phenylalanine) in A652(NR1) and A651(NR2B) also do not lead to constitutively open channels, double mutations [A652L(NR1) + A651L(NR2B) and $\mathrm{A} 652 \mathrm{~F}(\mathrm{NR} 1)+\mathrm{A} 651 \mathrm{~F}(\mathrm{NR} 2 \mathrm{~B})]$ significantly increase the constitutive open index to $\sim 46-56 \%$. Moreover, single mutations by substituting tryptophan (which has the "largest" side chain) to A652(NR1) and A651(NR2B) result in a constitutive open index of 10 and $30 \%$, respectively, whereas the homologous double mutation [A652W(NR1)+A651W(NR2B)] leads to a nearly fully constitutively open channel with an index up to $\sim 97 \%$ (Fig. $6 B$ ). The significant positive coupling energies $\left(\Delta \Delta G_{\text {int }}=1.57-3.34 \mathrm{kcal} / \mathrm{mol}\right)$ (Table 1) of these double mutations also indicate a strong cooperative interaction between A652(NR1) and A651(NR2B), well consistent with the proximity between A652(NR1) and A651(NR2B) demonstrated by the evident cooperative effect in the compensatory-charge double mutations. These findings altogether suggest an essential role of intersubunit configuration at the level of A652(NR1)A651(NR2B) in NMDA receptor activation-deactivation (see Discussion).

\section{Discussion}

The activation gate of the NMDA receptor is constituted by $\mathrm{M} 3 \mathrm{c}$ and is located in the external vestibule of the pore

We show that different single point mutations between S645 and I655 of M3c in NR2B (and also homologous residues in NR1) lead to constitutive opening of the NMDA receptor (Fig. 1). The currents through these "constitutively open channels" are readily blocked by external $\mathrm{Mg}^{2+}$ and MK-801. Also, MK-801 can no longer be trapped in these constitutively open channels (Fig. 2), 

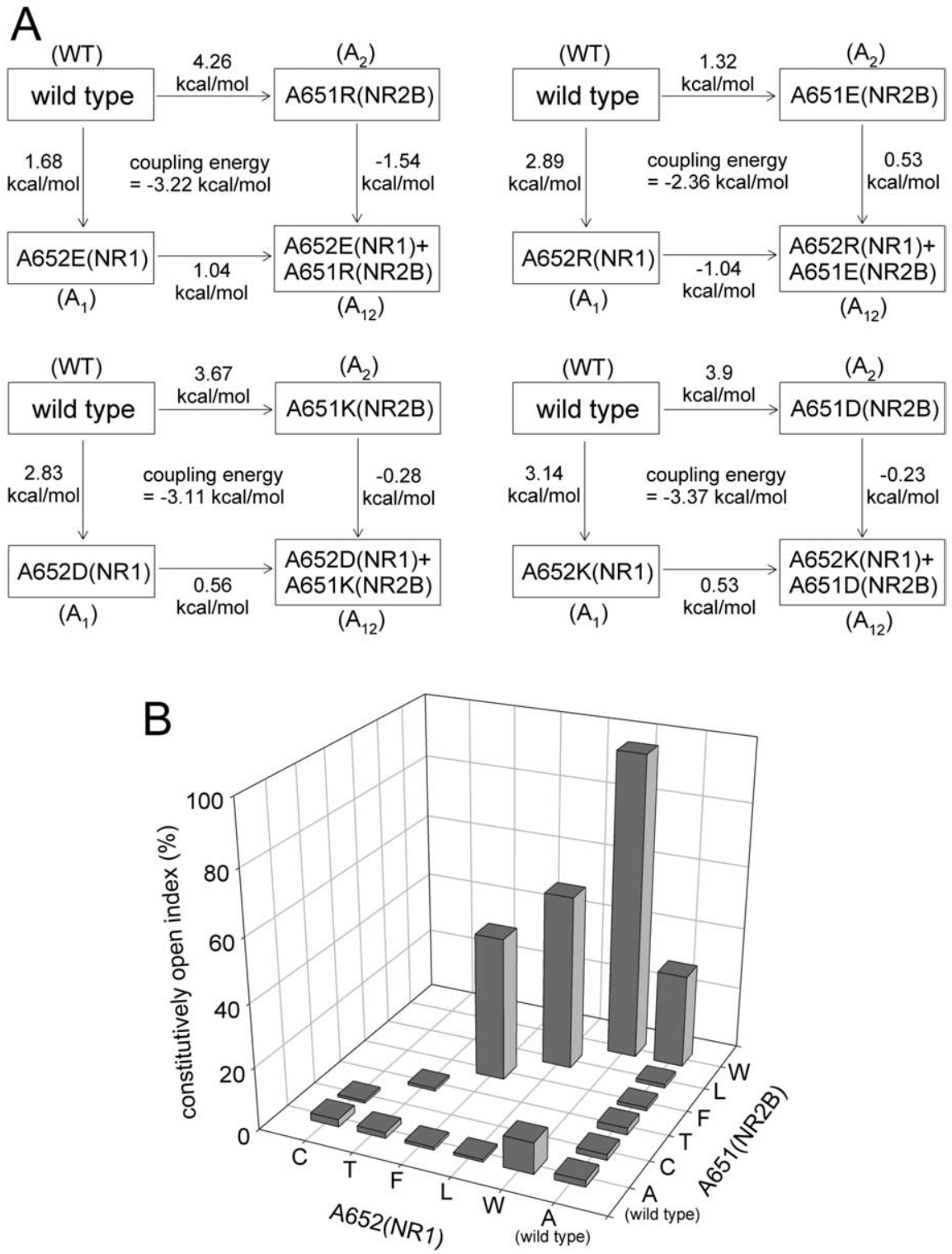

Figure 6. Thermodynamic double-mutant cycle analysis of different mutant receptors. $A$, For each channel, $\Delta \Delta G$ is calculated as that described in Results (Table 1). The sum of $\Delta \Delta G$ from wild type (WT) to $A_{1}$ and $A_{1}$ to $A_{12}$ equals the sum of $\Delta \Delta G$ from wild type to $A_{2}$ and $A_{2}$ to $A_{12}$, as required for a thermodynamic cycle. The sum of $\Delta \Delta G$ from wild type to $A_{1}$ and wild type to $A_{2}$, however, is evidently different from the sum of $\Delta \Delta G$ from wild type to $A_{1}$ and $A_{1}$ to $A_{12}$ or wild type to $A_{2}$ and $A_{2}$ to $A_{12}$ in all of the four cases presented here, indicating that the effects of the mutations on $\Delta \Delta G$ are not independent. $B$, Plot of the constitutive open index for single and double mutations by substituting A652(NR1) and A651(NR2B) with residues of different side-chain volumes (alanine, $\sim 15 \AA^{3}$ in the wild-type channel; cysteine and threonine, $\sim 38-43 \AA^{3}$; phenylalanine and leucine, $\sim 78-85$ $\AA^{3}$; and tryptophan, $\sim 115 \AA^{3}$ ).

consistent with the ideas that the foregoing residues are located in the external vestibule of the pore and are closely related to the activation gating control of the channel (see Introduction). In this regard, it is interesting to note that felbamate, the only approved anticonvulsant with significant gating-modification effects on NMDA receptors (Kuo et al., 2004), also binds to this region and acts as an "opportunistic" pore blocker with the "entrance" of its binding site undergoing a large gating conformational change (Chang and Kuo, 2007a,b, 2008). Moreover, MTSEA and MTSET modification of M3c (NR2B) is 500- to 1000fold faster in the activated than in the closed channels for the positions below A648 -A651 (Fig. 3C,D) (Jones et al., 2002; Sobolevsky et al., 2002a,b, 2007) (but see Beck et al., 1999). In contrast, the positions above A648-A651 (e.g., M654 and I655) are readily modified in both activated and closed channels. Most importantly, Figure 4 demonstrates that the NMDA concentration dependence of MTSET modification rate at $\mathrm{L} 643 \mathrm{C}$, a residue below A648-A651, is exactly superimposable on the NMDA concentration dependence of channel activation (opening probability of the channel). These findings indicate that the access of external MTS reagents and the permeating $\mathrm{Na}^{+}$to the channel pore is controlled by the same activation gate.

\section{A651(NR2B) and A652(NR1) of M3c constitute the critical part of the activation gate and ensure the essential intersubunit configuration for normal gating}

The significant negative coupling energies $\left(\Delta \Delta G_{\text {int }}=-2.36\right.$ to $\left.-3.37 \mathrm{kcal} / \mathrm{mol}\right)$ (Fig. 6A) of the compensatory-charge double mutations of A652(NR1) and A651(NR2B) by thermodynamic cycle analysis (Hidalgo and MacKinnon, 1995) indicate significant interactions between these two homologous residues in NR1 and NR2B. We have demonstrated that residues L643 and T647 of M3c in NR2B (and homologous V644 and T648 in NR1) constitute the binding ligands for felbamate (Chang and Kuo, 2008), suggesting that L643 and T647 approximately face the same side on the $\alpha$-helical M3c domain (Beck et al., 1999; Kashiwagi et al., 2002) and are both responsible for pore lining. The significant interactions between A652(NR1) and A651(NR2B) in compensatory-charge double mutations would then strongly argue that these two residues still line the pore and thus should be located close to the interphase of two different subunits if L643 and T647 in the same $\alpha$-helix are also in the pore. Consistently, single and double mutations at A652(NR1) and A651 (NR2B) demonstrate evident steric or voluminal effect on constitutive activation of the channel (Fig. $6 \mathrm{~B})$. Although single mutations to large side chains in A652(NR1) and A651 (NR2B) (both being A7 in the SYTANLAAF motif), such as A7L or A7F, do not lead to constitutively open channels, double mutations of A7 [A652L(NR1) + A651L(NR2B) and A652F(NR1)+ $\mathrm{A} 651 \mathrm{~F}(\mathrm{NR} 2 \mathrm{~B})]$ result in a substantial constitutive open index. Moreover, modification of A7 by external MTS reagents can happen only in the activated but not the resting channel and then markedly enhances NMDA currents as well as holds the channel constitutively open (Fig. $3 A$ ). These findings indicate that proper interactions between A7 and its local environment are critical for the activation-deactivation gating and that the distance between each two subunits at the A7 level is a critical determinant for the status of the NMDA receptor activation gate. The presumably very limited space to accommodate these critical residues in the resting channel, for example, may explain why alanines are se- 
lected and remain so highly conserved for this position (Fig. 7A). An approximately similar pattern of state-dependent modification of the SYTANLAAF motif has also been demonstrated for NR1 and NR2C (Sobolevsky et al., 2002a, 2007), and it seems that MTS modification of A7C in any subunit always lead to markedly enhanced constitutive opening of the channel (Beck et al., 1999; Jones et al., 2002; Yuan et al., 2005; Sobolevsky et al., 2007). However, the A7C mutations in NR1 and NR2A behave differently in response to partial glutamate site agonists (Jones et al., 2002). Also, there is probably a staggering alignment of $\mathrm{M} 3 \mathrm{c}$ by approximately four residues between subunits NR1 and NR2C. Moreover, the NMDA currents are markedly enhanced by MTS modification of only A7C and $\mathrm{A} 8 \mathrm{C}$ in NR1, but a similar effect is observed for A3C, N4C, A7C, and A8C in NR2C, with more $\mathrm{M} 3 \mathrm{c}$ residues in NR1 facing the central pore than in NR2C (Sobolevsky et al., 2002a,b, 2007). Thus, there may well be a different alignment of $\mathrm{M} 3 \mathrm{c}$ between NR1 and NR2A or NR2C, reminiscent of the findings that the tip of M2 is asymmetrically positioned between NR1 and NR2C (Kuner et al., 1996), although the M3c in NR2B seems to be well aligned to its homologous residues in NR1 in our case (Fig. 6) (Chang and Kuo, 2008). At any rate, it seems that the intersubunit distance at the level of A7 is well preserved as a common determinant of channel activation for all different subunits. It would be interesting to further characterize the differences in the local placement of M3c and related functional consequences for different subunits.

A652(NR1) and A651(NR2B) may mark the bundle-crossing point of the four

M3c helices

The pore region of the NMDA receptor is probably a minimal $\mathrm{K}^{+}$channel, which is "inserted" into a bacterial periplasmic protein in an "inverted" way (Kuner et al., 1996, 2003; Panchenko et al., 2001; Mayer and Armstrong, 2004). Identification of a prokaryotic ionotropic glutamate receptor with a $\mathrm{K}^{+}$selective pore (GluR0) (Chen et al., 1999) confirms this idea and strengthens the view that ionotropic glutamate receptors are structurally related to $\mathrm{K}^{+}$channels. Sequence alignment of the M3 segment of the NMDA or GluR0 receptors with the M2 (inner) segment of KcsA or the S6 segment of Shaker $\mathrm{K}^{+}$ channels (Fig. 7A) shows that A7 (A652 in NR1 and A651 in NR2B) in the NMDA receptor may correspond to A111 in KcsA and V478 in Shaker, the "bundle-crossing" points of those channels (Doyle et al., 1998; Hackos et al., 2002; Kitaguchi et al., 2004). This is consistent with our previous analysis that the in-

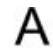

$\mathbf{P}$

M2/S6

KCSA 057 AOLITYPRALWWSVETATTVGYGDLYPVTLWGRLVAVVVMVAGITSFGLVTAALATWFVGRE 118 MthK 041 IEGESWTVSLYWTFVTIATVGYGDYSPSTPLGMYFTVTLIVLGIGTFAVAVERLLEFLIN-- 100 Shaker 424 SFFKSIPDAFWWAVVTMTTVGYGDMTPVGVWGKIVGSLCAIAGVLTIALPVPVIVSNFNYFY 485 GlURO 194 ----GVQNGMWFALVTLTTVGYGDRSPRTKLGQLVAGVWMLVALLSFSSITAGLASAFST-- 249 NR1 598 EDALTLSSAMWFSWGVLLNSGIGEGAPRSFSARILGMVWAGFAMIIVASYTANLAAFLVLDR 659 NR2A 596 GPSFTIGKAIWLLWGLVFNNSVPVQNPKGTTSKIMVSVWAFFAVIFLASYTANLAAFMIQEE 657 NR2B 597 GPSFTIGKAIWLLWGLVFNNSVPVQNPKGTTSKIMVSVWAFFAVIFLASYTANLAAFMIQEE 658 NR2C 607 GPSFTIGKSVWLLWALVFNNSVPIENPRGTTSKIMVLVWAFFAVIFLASYTANLAAFMIQEQ 668 M2 M3
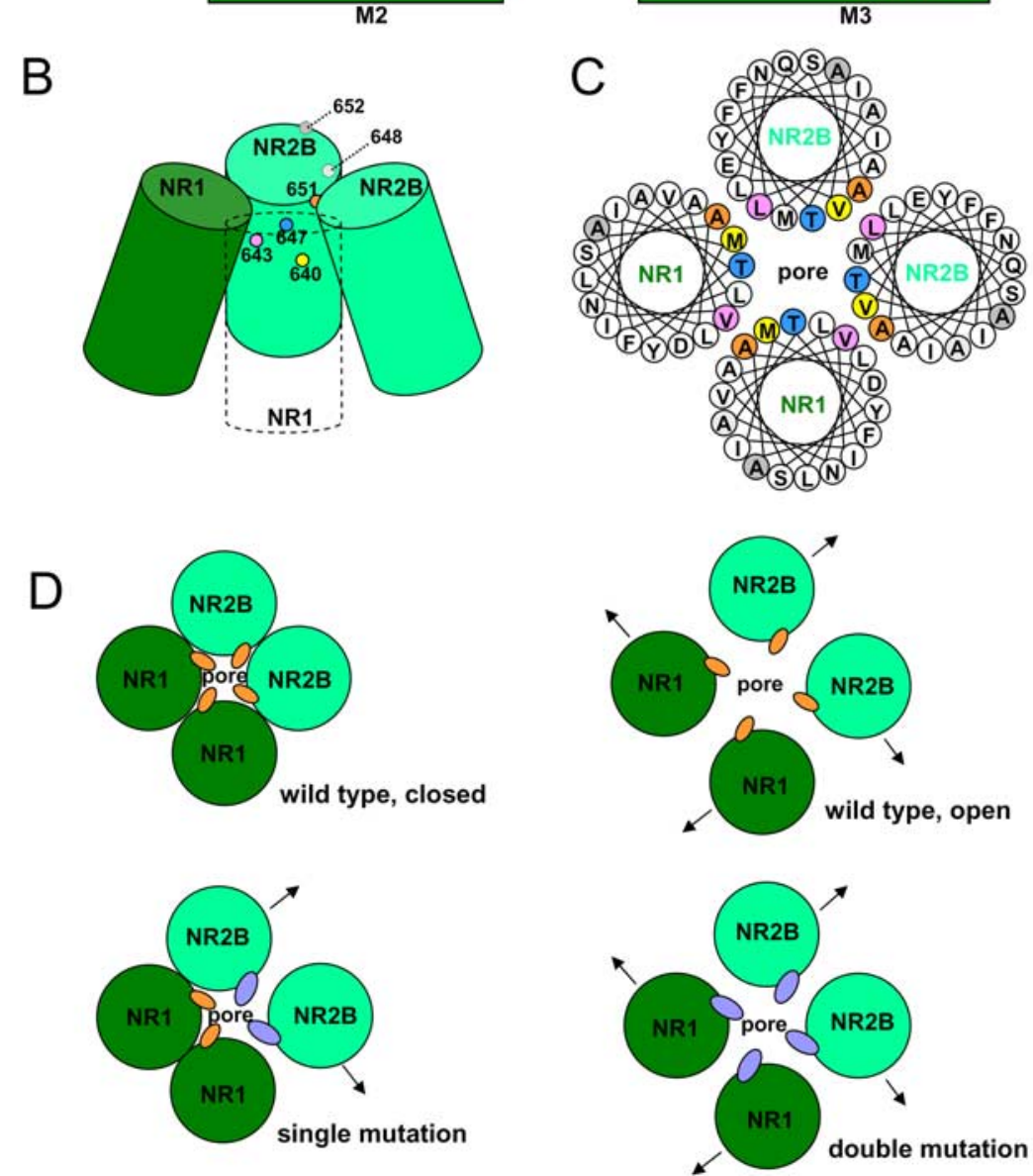

Figure 7. Molecular mechanisms underlying the activation gating control of the NMDA receptor. $A$, Amino acid sequence alignment for the pore-forming segments of structurally related channels. The sequences are shown for prokaryotic $\mathrm{K}^{+}$channels (KcsA and MthK), Shaker $\mathrm{K}^{+}$channel, prokaryotic ionotropic glutamate receptor (GluRO), and the NMDA receptor (NR1, NR2A, NR2B, and NR2 ( subunits). Amino acid numbers are given on both sides. The pore segment (P) and inner segment (M2/S6) in $\mathrm{K}^{+}$channels as well as M2 and M3 segments in the NMDA receptor are indicated. The highly conserved SYTANLAAF motifs in NMDA receptors are colored red. The bundle crossing (the activation gate) of $\mathrm{K}^{+}$channels is marked by an arrow. $\boldsymbol{B}$, Four M3 $\alpha$-helices from two NR1 and two NR2B subunits are arranged as a bundle (or a teepee) circling the NMDA channel pore with the top facing the extracellular side. We assume an NR1-NR1-NR2-NR2 orientation of subunits (Schorge and Colquhoun, 2003). For clarity, only the residues of one NR2B subunit are indicated by small circles. Residues V640 (yellow), L643 (pink), T647 (blue), and A651 (orange) in NR2B would face the same side and be responsible for pore lining. In this regard, residue A651(NR2B) is most likely situated at the "corner" or the narrowest intrapore region between two neighboring subunits. Conversely, residues A648 (light gray) and A652 (gray) in NR2B are located in the extrapore region. C, The M3 helical wheel diagrams of NR1 (M641-D658) and NR2B (V640 -E657) are shown as viewed from the extracellular side of the channel (the crossing angle of M3 helices is disregarded for simplicity). Residues V640, L643, T647, A648, A651, and A652 in NR2B (and their homologous residues in NR1) are colored yellow, pink, blue, light gray, orange, and gray, respectively. D, Schematic drawing of the activation gate at the level of A652(NR1)-A651(NR2B). Four small side chains (colored orange) of two A652(NR1) and two A651(NR2B) are well accommodated at the corner of the pore to ensure the appropriate intersubunit distance in the closed wild-type receptor. The pore at this level, or the "activation gate," is not wide enough for a smooth passage of hydrated $\mathrm{Na}^{+}$ions (top left). Binding of the agonists NMDA and glycine presumably would pull all four M3c helices outward to widen the pore at this level (to open the activation gate; top right). Single mutations by substituting amino acids with large side chains and/or the same charges (e.g., arginine, phenylalanine, leucine, or tryptophan; colored purple) to A652(NR1) or A651(NR2B) make two NR1 or two NR2B M3 chelices "repel" each other. The two interacting helices thus are moved outward from the pore axis, resulting in small constitutive opening of the activation gate (bottom left). Double mutations of both A652(NR1) and A651(NR2B) make all of the four M3c helices repel one another. The intersubunit distance, and consequently the pore at this level, is widened enough to allow a smooth passage of hydrated $\mathrm{Na}^{+}$ions even in the absence of NMDA and glycine and thus a constitutively open activation gate of the channel (bottom right). 
tersubunit distance at the A7 level is a common determinant of NMDA channel activation. A7 thus probably marks the narrowest (or the bundle-crossing) region of the external vestibule in the closed channel (Fig. 7 B, C). The other M3c mutations leading to constitutively open channels in the alanine scan (Fig. 1) presumably directly or allosterically change the intersubunit distance at this A7 level. This is reminiscent of the constitutive activation of the Shaker $\mathrm{K}^{+}$channel caused by mutations within the S6 activation gate region (Sukhareva et al., 2003). The ligand-binding core of the NMDA receptor consists of two domains formed by the N-terminal extracellular domain (S1) and the extracellular loop between M3 and M4 (S2) (Kuryatov et al., 1994; Laube et al., 1997; Anson et al., 1998; Furukawa and Gouaux, 2003; Chen et al., 2005; Furukawa et al., 2005). Because the conserved SYTANLAAF motif of the NMDA receptor is located at the end of M3c, which is directly connected to the S2 domain [the Helix F (Furukawa and Gouaux, 2003)] by a brief ( $\sim 30$ amino acids) post-M3 segment, via which the en bloc movement caused by ligand binding may be readily transduced. The diameters at the levels V644(NR1)-L643(NR2B) and T648(NR1)-T647(NR2B) of $\mathrm{M} 3 \mathrm{c}$ in the open channel are estimated to be $\sim 18$ and $\sim 20 \AA$, respectively (from $\alpha$-carbon to $\alpha$-carbon in the main chain) (Chang and Kuo, 2008). With an $\alpha$-helical secondary structure, the diameter at the level of A652(NR1)-A651(NR2B) then should be $\sim 22 \AA$ ( $\alpha$-carbon to $\alpha$-carbon) or $\sim 17 \AA$ (side chain tip to tip) in the open channel. If the diameter at this level should be $<7 \AA$ (the smallest diameter of an hydrated $\mathrm{Na}^{+}$ion) in the closed channel, there would be a $>10 \AA$ gating conformational change at the activation gate of the NMDA receptor, a figure slightly larger than that in $\mathrm{K}^{+}$channels (e.g., KcsA, MthK, and Kv1.2), in which diameters of $\sim 4-5$ and $\sim 12 \AA$ at the intracellular end were reported for the closed and open channels, respectively (Doyle et al., 1998; Jiang et al., 2002a,b; Long et al., 2005a,b).

\section{Effective opening of the activation gate requires movement of all four M3c helices}

The opening probability of NMDA channel is greatly increased only when all of the four agonist sites are occupied by appropriate agonists (e.g., two glutamates and two glycines), indicating that effective opening of the activation gate requires all of four M3c helices. Consistently, we have seen that single mutations of A7 into residues with large hydrophobic side chains (leucine or phenylalanine) lead to just negligible, and even single mutations into tryptophan (which has the largest side chain) lead to only small, constitutive channel opening (Fig. 7D). In contrast, when all four $\mathrm{M} 3 \mathrm{c}$ helices contain bulky substituting side chains at A7, there is a disproportionately enhanced constitutive opening in these double-mutant NMDA receptors. The arrangement and motion of the four $\mathrm{M} 3 \mathrm{c}$ helices at the A7 activation gate must be so designed that movement of only two subunits would have a disproportionately smaller effect than movement of all four subunits, and only when all M3c helices tend to decline a close apposition with their neighbors at the A7 level could the channel fully open, regardless of whether the movement is achieved by binding of glutamate and glycine or by steric hindrance and/or other repulsive forces between different M3c helices.

\section{References}

Anson LC, Chen PE, Wyllie DJ, Colquhoun D, Schoepfer R (1998) Identification of amino acid residues of the NR2A subunit that control glutamate potency in recombinant NR1/NR2A NMDA receptors. J Neurosci 18:581-589.
Antonov SM, Gmiro VE, Johnson JW (1998) Binding sites for permeant ions in the channel of NMDA receptors and their effects on channel block. Nat Neurosci 1:451-461.

Beck C, Wollmuth LP, Seeburg PH, Sakmann B, Kuner T (1999) NMDAR channel segments forming the extracellular vestibule inferred from the accessibility of substituted cysteines. Neuron 22:559-570.

Bruening-Wright A, Schumacher MA, Adelman JP, Maylie J (2002) Localization of the activation gate for small conductance $\mathrm{Ca}^{2+}$-activated $\mathrm{K}^{+}$ channels. J Neurosci 22:6499-6506.

Chang H-R, Kuo C-C (2007a) Characterization of the gating conformational changes in the felbamate binding site in NMDA channels. Biophys J 93:456-466.

Chang H-R, Kuo C-C (2007b) Extracellular proton-modulated poreblocking effect of the anticonvulsant felbamate on NMDA channels. Biophys J 93:1981-1992.

Chang H-R, Kuo C-C (2008) Molecular determinants of the anticonvulsant felbamate binding site in the $N$-methyl-D-aspartate receptor. J Med Chem, in press.

Chen GQ, Cui C, Mayer ML, Gouaux E (1999) Functional characterization of a potassium-selective prokaryotic glutamate receptor. Nature 402:817-821.

Chen PE, Geballe MT, Stansfeld PJ, Johnston AR, Yuan H, Jacob AL, Snyder JP, Traynelis SF, Wyllie DJ (2005) Structural features of the glutamate binding site in recombinant NR1/NR2A N-methyl-D-aspartate receptors determined by site-directed mutagenesis and molecular modeling. Mol Pharmacol 67:1470-1484.

Dingledine R, Borges K, Bowie D, Traynelis SF (1999) The glutamate receptor ion channels. Pharmacol Rev 51:7-61.

Doyle DA, Morais Cabral J, Pfuetzner RA, Kuo A, Gulbis JM, Cohen SL, Chait BT, MacKinnon R (1998) The structure of the potassium channel: molecular basis of $\mathrm{K}^{+}$conduction and selectivity. Science 280:69-77.

Dravid SM, Erreger K, Yuan H, Nicholson K, Le P, Lyuboslavsky P, Almonte A, Murray E, Mosley C, Barber J, French A, Balster R, Murray TF, Traynelis SF (2007) Subunit-specific mechanisms and proton sensitivity of NMDA receptor channel block. J Physiol (Lond) 581:107-128.

Flynn GE, Zagotta WN (2001) Conformational changes in S6 coupled to the opening of cyclic nucleotide-gated channels. Neuron 30:689-698.

Furukawa H, Gouaux E (2003) Mechanisms of activation, inhibition and specificity: crystal structures of the NMDA receptor NR1 ligand-binding core. EMBO J 22:2873-2885.

Furukawa H, Singh SK, Mancusso R, Gouaux E (2005) Subunit arrangement and function in NMDA receptors. Nature 438:185-192.

Hackos DH, Chang TH, Swartz KJ (2002) Scanning the intracellular S6 activation gate in the shaker $\mathrm{K}^{+}$channel. J Gen Physiol 119:521-532.

Hidalgo P, MacKinnon R (1995) Revealing the architecture of a $\mathrm{K}^{+}$channel pore through mutant cycles with a peptide inhibitor. Science 268:307-310.

Huettner JE, Bean BP (1988) Block of N-methyl-D-aspartate-activated current by the anticonvulsant MK-801: selective binding to open channels. Proc Natl Acad Sci USA 85:1307-1311.

Jiang Y, Lee A, Chen J, Cadene M, Chait BT, MacKinnon R (2002a) Crystal structure and mechanism of a calcium-gated potassium channel. Nature 417:515-522.

Jiang Y, Lee A, Chen J, Cadene M, Chait BT, MacKinnon R (2002b) The open pore conformation of potassium channels. Nature 417:523-526.

Jones KS, VanDongen HM, VanDongen AM (2002) The NMDA receptor M3 segment is a conserved transduction element coupling ligand binding to channel opening. J Neurosci 22:2044-2053.

Kashiwagi K, Masuko T, Nguyen CD, Kuno T, Tanaka I, Igarashi K, Williams K (2002) Channel blockers acting at $N$-methyl-D-aspartate receptors: differential effects of mutations in the vestibule and ion channel pore. Mol Pharmacol 61:533-545.

Kitaguchi T, Sukhareva M, Swartz KJ (2004) Stabilizing the closed S6 gate in the Shaker Kv channel through modification of a hydrophobic seal. J Gen Physiol 124:319-332.

Kohda K, Wang Y, Yuzaki M (2000) Mutation of a glutamate receptor motif reveals its role in gating and delta2 receptor channel properties. Nat Neurosci 3:315-322.

Kuner T, Wollmuth LP, Karlin A, Seeburg PH, Sakmann B (1996) Structure of the NMDA receptor channel M2 segment inferred from the accessibility of substituted cysteines. Neuron 17:343-352. 
Kuner T, Seeburg PH, Guy HR (2003) A common architecture for $\mathrm{K}^{+}$channels and ionotropic glutamate receptors? Trends Neurosci 26:27-32.

Kuo C-C, Liao S-Y (2000) Facilitation of recovery from inactivation by external $\mathrm{Na}^{+}$and location of the activation gate in neuronal $\mathrm{Na}^{+}$channels. J Neurosci 20:5639-5646.

Kuo C-C, Lin B-J, Chang H-R, Hsieh C-P (2004) Use-dependent inhibition of the $\mathrm{N}$-methyl-D-aspartate currents by felbamate: a gating modifier with selective binding to the desensitized channels. Mol Pharmacol 65:370-380.

Kuryatov A, Laube B, Betz H, Kuhse J (1994) Mutational analysis of the glycine-binding site of the NMDA receptor: structural similarity with bacterial amino acid-binding proteins. Neuron 12:1291-1300.

Laube B, Hirai H, Sturgess M, Betz H, Kuhse J (1997) Molecular determinants of agonist discrimination by NMDA receptor subunits: analysis of the glutamate binding site on the NR2B subunit. Neuron 18:493-503.

Liu Y, Holmgren M, Jurman ME, Yellen G (1997) Gated access to the pore of a voltage-dependent $\mathrm{K}^{+}$channel. Neuron 19:175-184.

Long SB, Campbell EB, Mackinnon R (2005a) Crystal structure of a mammalian voltage-dependent Shaker family $\mathrm{K}^{+}$channel. Science 309:897-903.

Long SB, Campbell EB, Mackinnon R (2005b) Voltage sensor of Kv1.2: structural basis of electromechanical coupling. Science 309:903-908.

Low CM, Lyuboslavsky P, French A, Le P, Wyatte K, Thiel WH, Marchan EM, Igarashi K, Kashiwagi K, Gernert K, Williams K, Traynelis SF, Zheng F (2003) Molecular determinants of proton-sensitive N-methyl-Daspartate receptor gating. Mol Pharmacol 63:1212-1222.

Mayer ML, Armstrong N (2004) Structure and function of glutamate receptor ion channels. Annu Rev Physiol 66:161-181.

Monyer H, Sprengel R, Schoepfer R, Herb A, Higuchi M, Lomeli H, Burnashev N, Sakmann B, Seeburg PH (1992) Heteromeric NMDA receptors: molecular and functional distinction of subtypes. Science 256:1217-1221.

Moriyoshi K, Masu M, Ishii T, Shigemoto R, Mizuno N, Nakanishi S (1991) Molecular cloning and characterization of the rat NMDA receptor. Nature 354:31-37.

Panchenko VA, Glasser CR, Mayer ML (2001) Structural similarities between glutamate receptor channels and $\mathrm{K}^{+}$channels examined by scanning mutagenesis. J Gen Physiol 117:345-360.
Perin-Dureau F, Rachline J, Neyton J, Paoletti P (2002) Mapping the binding site of the neuroprotectant ifenprodil on NMDA receptors. J Neurosci 22:5955-5965.

Qian A, Johnson JW (2002) Channel gating of NMDA receptors. Physiol Behav 77:577-582.

Schorge S, Colquhoun D (2003) Studies of NMDA receptor function and stoichiometry with truncated and tandem subunits. J Neurosci 23:1151-1158.

Sobolevsky AI, Koshelev SG, Khodorov BI (1999) Probing of NMDA channels with fast blockers. J Neurosci 19:10611-10626.

Sobolevsky AI, Beck C, Wollmuth LP (2002a) Molecular rearrangements of the extracellular vestibule in NMDAR channels during gating. Neuron 33:75-85.

Sobolevsky AI, Rooney LA, Wollmuth LP (2002b) Staggering of subunits in NMDAR channels. Biophys J 83:3304-3314.

Sobolevsky AI, Prodromou ML, Yelshansky MV, Wollmuth LP (2007) Subunit-specific contribution of pore-forming domains to NMDA receptor channel structure and gating. J Gen Physiol 129:509-525.

Sukhareva M, Hackos DH, Swartz KJ (2003) Constitutive activation of the Shaker Kv channel. J Gen Physiol 122:541-556.

Sunami A, Tracey A, Glaaser IW, Lipkind GM, Hanck DA, Fozzard HA (2004) Accessibility of mid-segment domain IV S6 residues of the voltage-gated $\mathrm{Na}^{+}$channel to methanethiosulfonate reagents. J Physiol (Lond) 561:403-413.

Thomas CG, Krupp JJ, Bagley EE, Bauzon R, Heinemann SF, Vissel B, Westbrook GL (2006) Probing N-methyl-D-aspartate receptor desensitization with the substituted-cysteine accessibility method. Mol Pharmacol 69:1296-1303

Xie C, Zhen XG, Yang J (2005) Localization of the activation gate of a voltage-gated $\mathrm{Ca}^{2+}$ channel. J Gen Physiol 126:205-212.

Yuan H, Erreger K, Dravid SM, Traynelis SF (2005) Conserved structural and functional control of $\mathrm{N}$-methyl-D-aspartate receptor gating by transmembrane domain M3. J Biol Chem 280:29708-29716.

Zuo J, De Jager PL, Takahashi KA, Jiang W, Linden DJ, Heintz N (1997) Neurodegeneration in Lurcher mice caused by mutation in delta2 glutamate receptor gene. Nature 388:769-773. 